\title{
Increasing fruit and vegetable intake in low-income minority children: African American mothers as experts on effective strategies and barriers to FV.
}

\author{
Shriver LH*, Buehler C, Causey S, Porter L \\ Department of Nutrition, School of Health and Human Sciences, University of North Carolina Greensboro, Greensboro, \\ North Carolina, USA
}

\begin{abstract}
Objective: Eating habits and taste preferences begin to develop early in life, yet little is known about self-efficacy and struggles related to promoting FV among young children directly from the maternal perspective. Poor diet quality, including low FV consumption, is associated with race/ ethnicity- and income-based health disparities in the U.S., with low-income African Americans reporting the lowest intakes. Thus, the purpose of this study was to explore parenting strategies that are perceived as effective for encouraging FV consumption among low-income African American mothers of young children in order to inform future interventions for this at-risk population.

Methods: Mothers of 3 to 5-year-old children from two Head Start programs in a southeastern state of the U.S. were recruited for focus group participation. Focus group transcripts were analyzed using a content analysis to identify main themes in relation to perceived effective practices, and key barriers and facilitators to encouraging children's FV consumption.

Results: Accessibility strategies were uniquely perceived as effective for encouraging fruit. Negotiating/coaxing, making available, and using rules were perceived as effective and unique practices for vegetables. Child openness and maternal FV preferences facilitated maternal efforts to encourage FV consumption. Mothers identified three primary barriers to FV consumption: 1) child's "difficult" temperament; 2) negative family influences, and 3) low maternal FV preferences/modeling.

Conclusions: Low-income African American mothers utilize a large number and variety of strategies to encourage children's FV intake. Mothers themselves can serve as a key source of tips/strategies for each other's social and educational support. Future research should examine specific strategies that emerged in the current study in relation to actual FV consumption in lowincome African American children.
\end{abstract}

Keywords: Fruit and vegetables, Parenting practices, Children, African American, Low-income.

Accepted on March 16, 2018

\section{Introduction}

Fruits and vegetables (FV) are nutrient-dense foods that are rich in dietary fiber, antioxidants, and bioactive compounds [1]. Consumption of FV has been associated with reduced risks of coronary heart disease, diabetes, certain types of cancer, and also healthier adult weight $[2,3]$. Given the wide range of potential health benefits, the dietary recommendations emphasize the importance of $\mathrm{FV}$ as the foundation of healthy eating [1]. Despite these guidelines, the current FV intakes are well below the minimum recommendations across all age groups, with even larger deficits in low-income and minority populations $[4,5]$.

About $40 \%$ of African American youth aged 12-19 are overweight or obese, compared to $31 \%$ of Non-Hispanic White and $38 \%$ of Hispanic White children [6]. Many of the racial/ ethnic and income health disparities, including the higher obesity prevalence among low-income and minority populations in the U.S., are associated with nutrition-related factors, such as lower diet quality [6-8]. While factors contributing to health disparities are multifactorial, African Americans report the lowest consumption of FV in the nation [9]. Thus, increasing dietary intake of FV and establishing healthy eating habits with adequate amounts of FV from an early age represents a promising strategy for improving long-term health outcomes of this at-risk population [10].

Parents have an important influence on children's nutritional status because they control what, when, and how much children eat [11]. Parents can influence children's long-term health because they have a unique opportunity to affect children's early taste preferences and promote preference for healthy foods early in life. Parents can do so using a variety of practices, such as ensuring that FV are available to the child at home or by regularly preparing family meals with FV [12]. The construct of parenting practices was theoretically formulated in parenting literature by Darling and Steinberg [13] and refers to specific behaviors through which parents perform their parental duties to obtain a particular goal within a broader emotional climate. However, parenting practices can also include non-goal-directed parental behaviors, such as gestures, changes in tone of voice, or the spontaneous expression of emotion [13]. 
In the nutrition context, the construct of parenting practices has referred to specific behaviors that may be associated with favorable dietary outcomes among children, such as reduced obesity risk or decreased consumption of energy-dense foods $[14,15]$. Several previous studies have investigated the nature of parenting practices used specifically in relation to children's FV consumption [16]. For instance, a study by O'Connor et al. [17] examined parenting strategies related to $\mathrm{FV}$ intake in a sample of mostly mothers of children enrolled in the Head Start programs. Mothers reported using strategies that made FV available and accessible to their young children and also reported engaging with their child to encourage FV consumption (e.g., told the child he/she would be strong and/or healthy). A "non-directive food parenting" cluster was identified and characterized by parents who used strategies focused on increasing FV accessibility and child engagement, with less emphasis on pressuring the child to the target food. This type of parenting was associated with a greater intake of FV among children compared to types that incorporated different strategies. African American mothers represented the largest proportion of this "non-directive food parenting" cluster in the sample (37.5\% vs. $31.8 \%$ Hispanic, and $30.7 \%$ Caucasian American). The findings from this study indicate specific parenting strategies may, in fact, increase FV intake among children. More importantly, the use of specific parenting strategies likely differs by race/ethnicity and other parental characteristics that have not been well examined in existing studies [18].

Strategies parents use in feeding situations are influenced by their beliefs, knowledge, and perceptions related to nutrition and health [14]. Based on the socioecological perspective [10], parental attitudes and perceptions are also influenced by broader factors, such as cultural identification, economic resources, and cultural and social traditions that shape parental use of various food-related parenting strategies [18,19]. A study of more than 3,700 parent-child dyads found that controlling food-related practices, such as pressuring the child to eat or restricting his/ her intake, was more prevalent among racial/ethnic minorities and those with a lower household income and education [18]. Previous research with African American parents also highlighted the importance of generational influences, a strong desire for close social support, and the emphasis on oral tradition in food-related interactions (i.e., passing along recipes and history while cooking which promotes cultural connection) [10,20,21]. Because culture impacts not only health-related perceptions, but also food choices and food preparation practices, the intersectionality of culture, race, and socioeconomic status, should be considered when evaluating food-related parenting behaviors among low-income African American parents [21].

The Social Cognitive Theory (SCT) is a framework for explaining behaviors in health-related research [22] and has been widely utilized in nutrition studies, including studies related to parental influences on children's FV intake [23]. According to this framework, self-efficacy for achieving the desired behavior is one of the key determinants of successful health behavior change [22]. Parental perceptions of effective strategies for encouraging consumption of FV in low-income minority families with young children have not been well examined in previous research. Thus, formative research on parental perceptions of what strategies work and what the key barriers and facilitators are for promoting FV among young children is warranted to fully understand the parental perspectives [24]. This enriched understanding is needed to inform health care professionals working with parents in primary care settings and to inform future intervention efforts for improving FV intakes among young economically disadvantaged African American children. The main purpose of this study was to conduct a qualitative exploration of specific parenting strategies that lowincome African American parents perceive as effective for encouraging FV consumption by their 3-5 year old children. The second purpose was to explore factors that represent important facilitators and barriers for African American parents to successfully encourage children's consumption of FV.

\section{Materials and Methods}

\section{Study design, participants and procedures}

This study was conducted in two large Head Start programs in Southeastern U.S. Participants were recruited at health screenings and using study flyers posted throughout the buildings. Flyers also were sent home in children's backpacks. The participant inclusion criteria were:

(a) Having a 3 to 5 -year old-child enrolled in the Head Start program.

(b) Self-identifying as a African American individual.

(c) $\geq 18$ years old.

(d) The primary person responsible for child feeding and

(e) Child not having any condition that required a special diet (e.g., diabetes).

Focus groups were scheduled in the Head Start centers during convenient times for parents. Transportation and/or childcare during the focus groups were available. Prior to participant recruitment and data collection, the University's Institutional Review Board approved the study protocol.

\section{Study procedures for focus groups}

Two trained research assistants (at least one self-identified as African American) conducted the focus groups that lasted 1-1.5 hours to facilitate a comfortable and trusting environment for the participants A semi-structured focus group guide was created by the research team. Two senior researchers with expertise in nutrition and parenting reviewed the guide, discussed and finalized the questions after deliberations with two trained research assistants. After providing informed consent, parents were asked a series of open-ended questions centered around:

(a) Strategies parents used and considered as effective for encouraging their child to eat FV.

(b) Strategies parents did not consider effective.

(c) Parental explanations/reasoning for why they thought specific strategies were effective/ineffective.

Parents were asked each question separately for fruits and vegetables because of children's differential taste preferences for FV [25]. Data were collected from multiple focus groups 
until saturation was reached [26]. The co-facilitator took notes and summary reports were generated, with the assistance of the main facilitator after each focus group to aid in the data analysis and interpretation.

\section{Data analysis}

Focus groups were transcribed and analyzed using a manual in-depth content analysis by four trained researchers. Concept mapping approach was utilized to develop a framework for parenting practices related to $\mathrm{FV}$ consumption. A six-step process was utilized to summarize the main themes that emerged during the focus groups in relation to parenting practices and children's FV consumption:

(1) Recognition and labeling of researchers' preexisting expectations.

(2) Mark-up of statements in transcripts and note taking.

(3) Assignment of codes to text segments about parenting strategies that were:

(a) Perceived as effective for fruit.

(b) Perceived as effective for vegetables.

(4) Generation of a concept map for parenting strategy clusters perceived as effective for fruit and for vegetables.

(5) Discussion of each cluster, resolution of disagreements, and labeling of clusters as a parenting practice and

(6) Assignment of codes to focus group transcribed text segments that were perceived by parents as facilitators/enabling factors and/or barriers/constraining factors.

\section{Results}

A total of seven focus groups were completed during the study. All participants $(n=29)$ were mothers, except one who was a grandmother (the term "mothers and maternal" will be used from now on). The discussion generated several themes around parenting practices that mothers perceived as effective for encouraging the child to eat fruit and vegetables. Multiple factors that either facilitated or impeded maternal efforts to encourage fruit and vegetable consumption among young children also emerged. The main emerged themes related to effective parenting practices are presented in Figure 1. Representative quotes for each specific perceived effective practice for either fruit or vegetables are presented in Table 1.

\section{Perceived effective parenting practices for encouraging fruit}

Using the concept matching technique, we identified seven parenting practices, each evidenced by multiple strategies, that mothers perceived as effective when encouraging their child to consume fruit:

(a) Making fruit accessible: This practice was evidenced by maternal statements about having fruit ready for child to eat (e.g., having fruit reachable; choosing restaurants with fruit on menu).

(b) Presenting fruit: This practice was evidenced by statements about the way fruit was prepared for the child (e.g., serving the fruit with something else the child likes; offering fruit in the form the child prefers).

(c) Engaging the child in active learning with fruit: This practice emerged from statements about mothers teaching

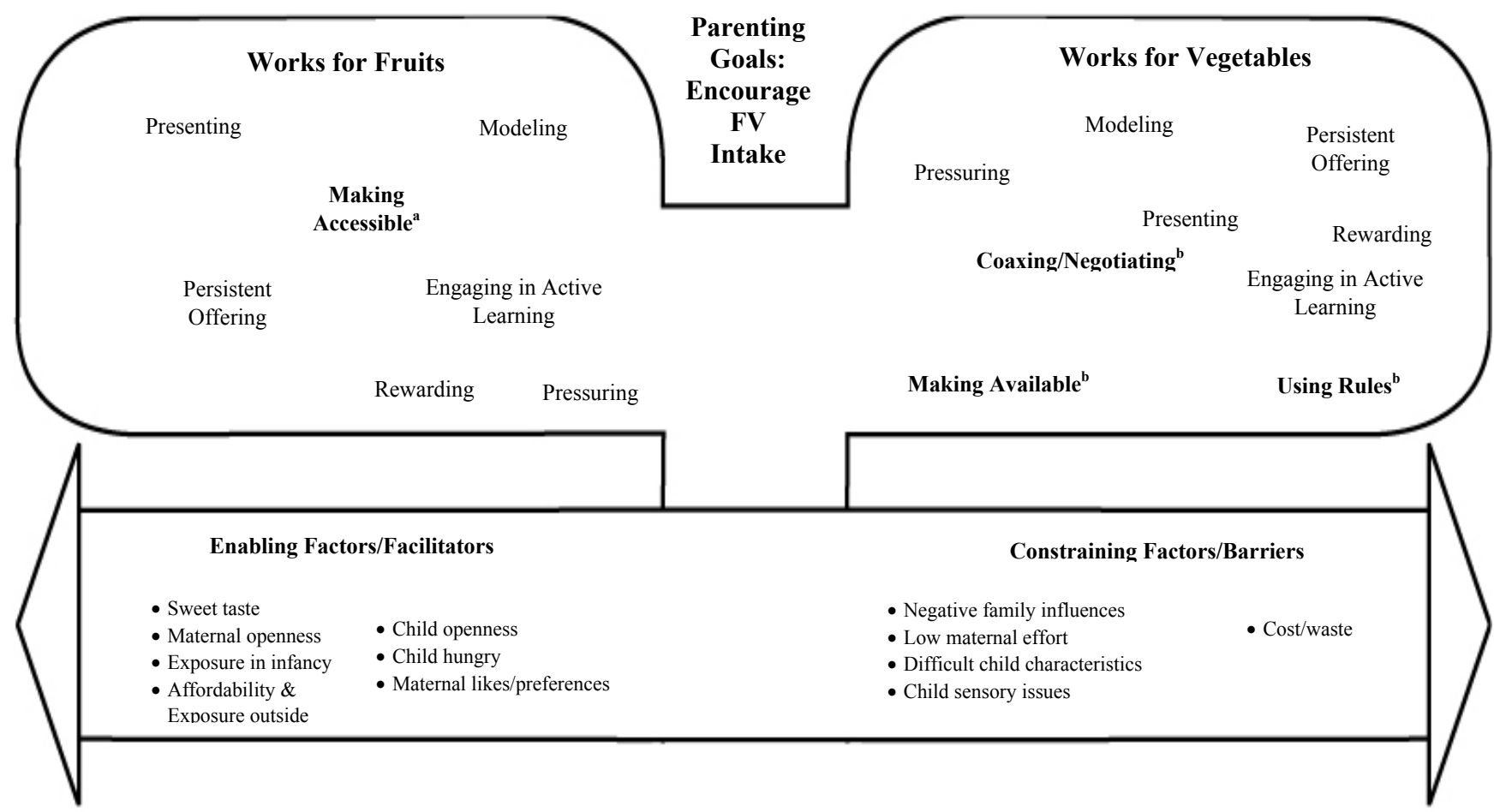

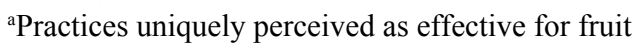

${ }^{\mathrm{b}}$ Practices uniquely perceived as effective for vegetables

Figure 1. Perceived effective maternal practices, facilitators and barriers for encouraging fruits and vegetables among 3 to 5-year-old. 
Citation: Shriver LH, Buehler C, Causey S, et al. Increasing fruit and vegetable intake in low-income minority children: African American mothers as experts on effective strategies and barriers to FV. J Child Adolesc Health. 2018;2(1):13-21.

Table 1. Representative quotes of parenting practices for fruit and vegetables perceived as effective by African American mothers of 3-5 years old children.

\begin{tabular}{|}
$\begin{array}{c}\text { Effective Practices } \\
\text { for Fruit }\end{array}$ \\
Making Fruit Accessible: \\
\hline $\begin{array}{c}\text { "You know I leave the fruit out... I put them in a bowl, but them on a table and you } \\
\text { know they'll eat it and my son will just grab... you know ask his sister or I to peel it." } \\
\text { "The bowl of fruit that I sit on the table because they look to that quicker than they } \\
\text { do anything else." } \\
\text { "I guess if you can see it, its more tangible where as if you don't think about it or } \\
\text { don't see it. Out of sight, out of mind..." } \\
\text { "...that's why I keep it in the refrigerator, if they want it they can go get grapes, } \\
\text { oranges, bananas, apples." } \\
\text { Presenting Fruit: } \\
\text { "So, I can pair almost anything with cheese, I don't care what it is and it'll work." } \\
\text { "I let them have their own little rind." } \\
\text { "They love dipping the fruit in the caramel... peanut butter..." } \\
\text { "I would blend it maybe make it into a smoothie make it some old school put some } \\
\text { yogurt in there some ice." } \\
\text { "We freeze small things like grapes and small strawberries, so they can go in there } \\
\text { and get something." }\end{array}$ \\
Engaging in Active Learning with Fruit:
\end{tabular}

"This is a kiwi, this is a mango, this is from there and the origin and tell her from where, how it gets grown because she'll ask me all of those things and that how I developed with her, like getting exposed to it. That's how I do it."

"I have to go through the whole education thing but that was the method I did, just try it, something I'm not really familiar...trial and error thing."

"I would cut it open, and explore it, we'd have to explore it, probably um and after we explore it and all the textures..."

\section{Persistent Offering:}

"I keep introducing it...and by repetition you know they'll end up trying it" "I try and get one that she does know and one she doesn't know so if she doesn't know then she has one..."

"When they want something really sweet I try to find sweet fruit to replace it" "I think it's all about when they want to eat it or not want to eat it, you know it's on them I guess sometimes they eat it sometimes they don't so I don't know... [it depends on] how hungry, how they feeling..."

"See I started early [offering fruit], I started like me first. What I ate, he ate and then when you know when your child is like four months, five months."

\section{Modeling Fruit Consumption:}

"I eat it and he usually eats whatever I'm eating, because see they all look at it and go "ooh, I don't want it" and they can see mommy sitting there enjoying it and if you don't want it that when I tell them I'll eat it, and I get to eating it and they say, "ooh ma, can I have some?" and they all get into my plate, next thing you know my whole plate gone and they all want they own plate."

"....It always tastes better off mommy's plate."

".... She'll eat it because I ate it...likely all of them [children] will come and trickle around."

"I think also from seeing you know mommy or grandma eating it, you know."

\section{Pressuring:}

"I'm like okay no Play station 3 and they like okay l'm gonna eat like three of these." "To me it's just a warning but l've done it though and it has worked."

\section{Rewarding:}

"Praise and compliments goes very far with mine."

\section{Effective Practices \\ For Vegetables

Presenting Vegetables:

"Broccoli chicken casserole...um just adding it in there."

"I put broccoli in macaroni and cheese."

"Cold but if it's warm and its seasoned they like that."

"I think most kids like spaghetti and stuff and I think that's a good way to add like a vegetable in that."

"Put the peas in with the mashed potatoes for them to eat it and even now I still have to do stuff like that for them."

"We started eating spinach...spinach dip uses the vegetables....and the vegetable dip to give it season... They love it."

"I'll throw in something new like a lima bean it could be some carrots."

\section{Coaxing or Negotiating}

"But you gotta have a little bit you just at least have a little bit so by theory is that if you 've tried it you can actively say I don't like it"

"I'll be like l'll take a bite, you take a bite. If we go back and forth with the bites he still ate at least half of it."

"Taste it. He's never ever had it before. He gotta taste it. Well just, just nibble it, just see I'm not saying you gotta eat the whole piece but if I give you just a sliver or piece you gotta taste it. He tells me its nasty it's over, I'm not gonna force."

\section{Making Vegetables Available:}

"If you don't buy it they can't have it."

"...Whenever we do a cookout or something, they [my children] find it really coo that a lot of people go to these vegetable platters first..

"...And I stay consistent.... When I find out what my son like, that's what I have."

\section{Engaging in Active Learning with Vegetables:}

"I'm like an educator"

"But it's good for your heart so I'm always telling my children, I'm like the beets are good for your heart and your blood ..."

"I was telling I got them to eat cauliflower by telling them it looks just like broccoli, it's just white broccoli so once they tried it they were like okay it's no big deal."

"He likes Iron Man so if I tell him if he eats them he'll be strong like Iron Man."

\section{Modeling Vegetable Consumption:}

"I eat salads, tomatoes and stuff like that another thing that they eat because I eat it..."

"I'm a collard queen and so they have eaten collards"

"I would get probably get the ringleader in, that's the sister, their older sister, whatever she does they do..."

was like okay well you gotta eat some more vegetables. Or you wanna be big and strong? He likes Spiderman. Spiderman eat all his vegetables and his fruit/eat the fruit and vegetables."

\section{Rewarding}

"They may want a scoop of ice cream. I have to get what I want in order for them to get what they want.."

"...can have dessert afterwards."

"I'll give you whatever you want after this [eating vegetables]."

\section{Pressuring}

"If I do like maybe a hand full of vegetables like corn, green beans, broccoli, if you eat half of it, we can still be friends you know?"

"..You can't leave the table until at least half of it is gone."

\section{Using Rules:}

"If you're not hungry l'll put it up for later, I'm not going to fix you an alternative, so you know you can either eat your food."

"I'm not listening two hours from now, "I'm hungry," "You know when we were eating, you didn't eat your stuff."

"..When we get pizza, you gotta have salad" 
the child something about the fruit (e.g., letting the child experience the fruit with his/her senses; learning about health benefits of fruit.

(d) Persistent offering: This practice was evidenced by mothers repeatedly offering the same fruit or regularly introducing new fruit.

(e) Modeling fruit consumption: This practice was evidenced by different types of modeling strategies ranging from maternal modeling or using other family members for modeling. The last two practices emerged less often than others; some mothers indicated they used.

(f) Pressuring: Evidenced, for example, by not offering drinks until the fruit is eaten; and finally.

(g) Rewarding: Evidenced by statements about offering the child candy or dessert for eating the fruit.

\section{Perceived effective parenting practices for encouraging vegetables}

Eight effective practices related to vegetables emerged during the focus groups:

(a) Presenting vegetables: This practice was evidenced by a number of strategies mothers used to prepare/serve vegetables based in the child's likes (e.g., cold versus hot; mixed it with rice).

(b) Coaxing or negotiating: Evidenced by strategies designed to encourage, but not overly pressure, the child to eat the target vegetable (e.g., verbally encouraging to take a bite).

(c) Making vegetables available: This practice was evidenced by mothers having a variety of vegetables at home or serving vegetables at special events (e.g., birthday parties).

(d) Engaging the child in active learning with vegetables: This strategy was evidenced by mothers teaching the child something about the vegetables (e.g., reading about vegetables; teaching shapes while eating vegetables.

(e) Modeling vegetable consumption: As evidenced by mothers eating vegetables in front of the child or having someone else model vegetable consumption to the child.

(f) Rewarding: As evidenced by mothers offering cookies or TV time.

(g) Pressuring: This practice was evidenced by a variety of behaviors, such as withholding drinks, not allowing the child to leave the table); and finally.

(h) Using rules: Evidenced by mothers enforcing established rules for situations when the child does not want to eat the vegetable (e.g., not making anything different to eat for the child).

\section{Perceived facilitators and barriers to encouraging FV consumption}

Mothers discussed several topics that emerged as potential facilitators for encouraging FV consumption among children:

(a) Sweet taste. (b) Maternal openness/flexibility (e.g., giving the child FV choices).

(c) Maternal FV taste preferences.

(d) Child openness/flexibility; child being open to trying new foods.

(e) Child being hungry when offered FV and

(f) FV affordability (e.g., having WIC vouchers; child being exposed to some FV in Head Start.

Representative quotes for the specific facilitators are presented in Table 2.

Several constructs emerged as barriers or constraining influences on child FV consumption:

(a) Child with a difficult temperament (e.g. a picky eater, stubborn, refusing unfamiliar things).

(b) Negative family influences, which included both personal and environmental influences (e.g., mother not liking vegetables herself; siblings not liking FV.

(c) Low maternal effort and/or flexibility (e.g., maternal unwillingness to peel fruit for the child; maternal unwillingness to try new foods; lack of skills to prepare FV).

(d) Child sensory issues (e.g., child refusal due to appearance and/or texture (i.e., messy) and

(e) FV cost (e.g., concerns about FV cost, waste).

Representative quotes for the barriers that emerged from the focus groups are presented in Table 2.

\section{Discussion}

The purpose of this study was to examine parenting practices that are perceived as effective for encouraging FV among young low-income African American children. Our study fills an important gap in the current literature on perceived efficacy of maternal practices related specifically to $\mathrm{FV}$, and also on factors that may be enabling or impeding maternal efforts. While previous studies have examined parenting constructs in relation to children's FV intake [16,17,27], a paucity of research has focused on in-depth examination of perceived effectiveness of FV strategies from the maternal perspective. Because interventions that successfully promote FV among preschoolers in general are scarce [28], the in-depth input and unique experiences of the low-income African American mothers in the current study can inform the design and content of future programs.

Mothers in the current study discussed a variety of both, effective and less effective strategies, for encouraging their child to eat FV from their unique perspectives, influenced by their family backgrounds, living arrangements and other factors. As a group, they were very creative in their efforts to promote FV on a daily basis. We found that some parenting practices were uniquely perceived as effective for encouraging fruit, but did not emerge as perceived effective practices for vegetables. Previous research has consistently reported that individuals tend to have greater intakes of fruit over vegetables across age, gender and 
Citation: Shriver LH, Buehler C, Causey S, et al. Increasing fruit and vegetable intake in low-income minority children: African American mothers as experts on effective strategies and barriers to FV. J Child Adolesc Health. 2018;2(1):13-21.

Table 2. Perceived facilitators and barriers to encouraging FV intake among low-income African American mothers of 3-5-year children.

\begin{tabular}{|c|c|}
\hline Constraining Factors/Barriers & Enabling Factors/Facilitators \\
\hline - $\quad$ Child with difficult temperament & Sweet taste \\
\hline - $\quad$ Negative family influences (i.e., sibling) & Mother's openness/flexibility \\
\hline - Low maternal effort/flexibility & Early exposure (i.e., infancy) \\
\hline - $\quad$ Child sensory issues (i.e., taste, appearance) & Child openness \\
\hline \multirow[t]{2}{*}{ - Cost/waste } & - $\quad$ Child being hungry \\
\hline & - Affordability and exposure outside of home \\
\hline Representative Quotes & Representative Quotes \\
\hline $\begin{array}{l}\text { "She is determined... I'm not going to eat it." } \\
\text { "Cause he's picky." }\end{array}$ & $\begin{array}{l}\text { "She loves grapes, strawberries, apples, bananas, peaches, just about any fruit } \\
\text { that l've tried and bought...u think she'd like it as long as its sweet and she likes } \\
\text { the taste }\end{array}$ \\
\hline $\begin{array}{l}\text { "Cause, either someone else is, cause his little cousin you know he don't eat this and } \\
\text { he don't eat that, and he don't eat." }\end{array}$ & "I won't like force him because I feel like well okay, he's not ready." \\
\hline \multirow[t]{2}{*}{ "If she sees and the older sibling looks at it like "mm" she'll feel weird about it too." } & "You gotta respect how they feel." \\
\hline & "I let them choose which fruit or vegetable..." \\
\hline \multirow{2}{*}{$\begin{array}{l}\text { "I don't like stuff crunching in my mouth other than and apple or carrot or some lettuce } \\
\text { that's why I can't do bell peppers, that cringe." }\end{array}$} & "I expose mine very young, very young, um like variety of things." \\
\hline & \multirow{2}{*}{$\begin{array}{l}\text { "She's open to trying anything... She tried a peach and tore it up...bananas and } \\
\text { strawberries... we've had some kiwi I think...yeah she's open to anything." }\end{array}$} \\
\hline \multirow{2}{*}{$\begin{array}{l}\text { "I think a lot of reasons why my not eat vegetables more is because I don't know too } \\
\text { many dishes." }\end{array}$} & \\
\hline & \multirow{2}{*}{$\begin{array}{l}\text { "I've learned with my four-year-old, you can't make them eat they'll tell you when } \\
\text { they're hungry." }\end{array}$} \\
\hline "I think it was just the way it looked like the little seeds." & \\
\hline "He said it was a tree and he wasn't eating a tree." & \multirow{3}{*}{$\begin{array}{l}\text { "have to change yourself if you want your kids to [eat FV]" } \\
\text { "I don't know if it came from Head Start with him eating strawberries." }\end{array}$} \\
\hline "Green stuff she turns her nose up any green she doesn't like it." & \\
\hline $\begin{array}{l}\text { "Mine like blueberries too, but it's expensive } \\
\text { "I'm not gonna you know cook something I know they're not going to eat." }\end{array}$ & \\
\hline
\end{tabular}

socioeconomic groups $[4,9,28]$. This is not surprising because children's natural hesitancy to try unfamiliar foods, coupled with the bitter/tart tastes of many vegetables, makes it more difficult for parents to encourage vegetable consumption [29]. Mothers in our sample clearly expressed having less difficulty encouraging fruit than vegetables, with sweet taste of fruit often noted as the main reason. The participants reported difficulties only when encouraging a less commonly consumed fruit with a unique appearance, such as papaya or kiwi (i.e., fuzziness, seeds).

The key effective practices for encouraging fruit consumption in our sample were making fruit accessible and presenting it in ways the child preferred. These results are consistent with previous studies that identified accessibility as a significant correlate of children's FV consumption [30]. Other practices, such as modeling and being efficacious, were also perceived as effective, but were much less central than strategies that increase child's access to fruit or the fruit's attractiveness. Our findings about the importance of accessibility for fruit consumption among young African American children are important because most existing research has investigated parenting practices with FV combined, not as separate food groups [17,31].

Accessibility did not emerge as an effective parenting practice related to vegetables in the current study. This finding may be due to children having lower taste preferences for vegetables over fruit, and thus, increasing accessibility of vegetables alone without using other strategies may not be sufficient for encouraging children to actually consume vegetables. Foodrelated behaviors are tied to cultures and basic socialization processes within families [20,21] so it is also possible that this finding is due to cultural and family traditions related to vegetables in African American families [20]. Mothers in the current study focused their attention largely on cooked vegetables and discussed offering vegetables as part of larger lunch/dinner meals, with very few making comments about their children eating raw vegetables. This observation from the focus groups is consistent with our previous work with African American mothers of preschoolers, where children themselves expected vegetables to be part of a cooked meal [20]. Thus, it is likely that strategies for increasing accessibility of vegetables throughout the day to their children (i.e., raw vegetables for a snack) were not perceived as relevant by the mothers in our sample because most of the vegetables in their families are served cooked and as part of family meals. Our findings further reinforce the notion that fruits and vegetables are unique food groups with different tastes and textures, with diverse cultural and family traditions that ultimately influence their consumption [25].

In our study, three unique practices were perceived as effective for vegetables, with negotiating/coaxing being the most central, whether it meant talking the child into taking a few bites or just placing the vegetable in his/her mouth without having to eat it. A couple of previous studies [32,33] have suggested that "encouragement through negotiations" and "physical prompting and bargaining" were associated with positive dietary habits, including greater FV consumption. The finding that mothers utilized negotiating frequently and found it effective is promising in the light of previous studies where parents were shown to quickly label their children as "picky eaters" without providing adequate number of necessary exposures for the child to get familiar with the new fruit or vegetable. Because between 8-15 repeated visual and taste exposures are needed in order for children to acquire preference for a new food [34], coaxing reported in our study represents a potentially effective way to continue exposing children not only to visual appearance of vegetables, but also to the different tastes and textures.

Along with negotiating, presenting vegetables in the form/shape children prefer emerged as a core perceived effective practice in our study. Ranging from mixing different vegetables together to 
preparing casserole dishes, the number and variety of strategies that mothers used to serve vegetables to their children based on their likes and dislikes was impressive and indicated their knowledge and experience with trying to encourage their child to eat vegetables in the past. Parental self-efficacy has been identified as one of the important determinants of successful interventions aimed at improving children's nutrition-related behaviors [35]. Our findings suggest that mothers in our sample were confident and had in-depth knowledge of their children's preferences as they have reported trying a variety of presentation strategies to encourage their children's intake of both, fruit and vegetables.

The importance of parental modeling for children's eating behaviors has been well documented in research [36]. While modeling emerged during the focus groups, it was not discussed as one of the perceived effective practices. Instead, many mothers admitted their own vegetable intake was low, shared their dislikes for some vegetables, and discussed negative childhood experiences related to "having to eat vegetables." Parental intake and preference of FV have been identified as critical correlates of children's FV consumption in previous research [30]. Low maternal taste preferences for vegetables and lack of parental openness/willingness to trying new vegetables emerged as main barriers to encouraging children's vegetable consumption in our sample.

A majority of the mothers viewed pressure as relatively ineffective for encouraging their child to eat FV. These perceptions were based on the experiences with their own child, often stating "I tried to make him/her eat it" or "guilt him/her into eating it," but in most cases, mothers acknowledged that such strategies failed to achieve the target behavior. Our analyses also indicated that the use of pressure was attempted much more frequently with vegetables compared to fruit. The maternal perceptions of pressure in our study are consistent with previous quantitative research that found controlling strategies to be negatively related to FV consumption and largely ineffective in the promotion of children's long-term increased FV taste preferences [31,14].

One of the main barriers to FV promotion that emerged in the current study was having a child that was a "difficult child/picky eater." Research shows that food neophobia and food enjoyment are important correlates of children's FV consumption, with food neophobia potentially predicting intake of FV [37,38]. For instance, a study of 399 young African American and Non-Hispanic White children found that being a "picky eater" predicted not only a lower vegetable but also lower fruit intake compared to "non-picky eaters" [36]. Mothers in our sample discussed a variety of scenarios to illustrate their children's pickiness and their own frustration when feeding them. The examples ranged from the child only "sticking with what he/she knows" to "he/she is particular." Given the known differences between food neophobia and picky eating [39], our findings indicate that mothers of preschoolers may greatly benefit from nutrition education that targets these constructs from both nutrition and parenting/child development perspectives. The targeted education efforts would help mothers not only differentiate between food neophobia and picky eating, but it may also provide mothers with practical tips and strategies to manage feeding situations effectively while maintaining overall positive feeding environment [38].

The current study directly responds to the consensus report of food parenting experts that have called for greater utilization of qualitative methods to increase our understanding of the breadth of food parenting practices and parental motivations to use them [24]. Our study also challenges the use of labels such as parenting "practices" and "strategies" that have been utilized in previous research. Based on our findings, some parenting "practices" should be reconsidered given what we have learned from mothers in our focus groups. Although making fruit accessible or engaging the child in active learning have been previously identified as "practices" [16], mothers in our study reported multiple strategies under each "practice." Therefore, we argue that each "parenting practice" is evidenced by a variety of strategies that share the same feeding goal. The identification of these strategies will support future prevention work by offering a large "bag of tricks" from which parents can pick and utilize those strategies that are consistent with their culture, traditions, and social backgrounds $[21,10]$.

\section{Limitations}

Our study uniquely examined perceived effectiveness of feeding strategies used to encourage FV among low-income African American preschoolers. Mothers served as key and direct informants on which specific strategies work from their firsthand experiences. Our approach generated rich qualitative data that allowed for in-depth examination of maternal perceptions related to encouraging FV consumption; however, several study limitations must be noted. First, the findings come from selfreported data only; no observations of actual practices related to FVs were conducted. Second, mothers in our sample might have had a greater interest in nutrition and/or health than non-participants. Third, significant efforts were made to create a comfortable and trusting environment during the discussions; however, it is possible that some mothers did not feel comfortable talking about their child. Finally, children's FV consumption was not assessed because the study's aim was to examine parental perceptions related to FV rather than actual intake.

\section{Conclusion}

The current study highlights the large number and variety of strategies utilized by low-income African American mothers for encouraging FV among their 3 to 5-year-old children. Ranging from presenting the FV in creative ways to utilizing siblings as role models, our findings show that mothers are valuable key informants and their input on FV promotion is critical and warranted in both future research and practice. The findings indicate that only few mothers utilize multiple strategies within and across various parenting practices. However, mothers were eager to share ideas and strategies during the focus groups. Thus, providing social support opportunities would be useful in broadening mothers' repertoire of FV-related feeding behaviors, particularly for those mothers who use several strategies but only a couple of practices to encourage their children's FV consumption. 
Citation: Shriver LH, Buehler C, Causey S, et al. Increasing fruit and vegetable intake in low-income minority children: African American mothers as experts on effective strategies and barriers to FV. J Child Adolesc Health. 2018;2(1):13-21.

The current study highlights the complex nature of FV promotion in early childhood and the many challenges parents face, especially when trying to encourage vegetable intake, among young children. Our findings point out that successful encouragement of FV in early childhood goes well beyond the availability and cost of FV. Successful encouragement of FV includes parental knowledge of the child's specific likes and dislikes, preparation skills to try different presentation of $\mathrm{FV}$, family support as well as having efficacy skills to know when, how, and whether the child is ready to try a new fruit or vegetable. Pediatricians and other health care professionals working with low-income families with young children serve as an important source of advice and support at the primary prevention level. Future research is warranted to establish links between the strategies that emerged in our study and reported consumption of FV among low-income African American parents and their young children.

\section{Acknowledgments}

This study was funded through the Research Excellence Award from the Office of Research in the School of Health and Human Sciences, University of North Carolina Greensboro. We would like to thank the parents who participated in the study and Head Start administrators and staff for their support.

\section{References}

1. USDA. Dietary guidelines for Americans. US Department of Health and Human Services, Washington D.C., USA. 2015;pp:1-114.

2. Ledoux TA, Hingle MD, Baranowski T. Relationship of fruit and vegetable intake with adiposity: A systematic review. Obesity Reviews. 2011;12:143-50.

3. Boeing H, Bechthold A, Bub A, et al. Critical review: Vegetables and fruit in the prevention of chronic diseases. Eur J Nutr. 2012;51:637-63.

4. Lorson BA, Melgar-Quinonez HR, Taylor CA. Correlates of fruit and vegetable intakes in US children. J Am Diet Assoc. 2009;109:474-8.

5. Di Noia J, Byrd-Bredbenner C. Determinants of fruit and vegetable intake in low-income children and adolescents. Nutr Rev. 2014;72:575-90.

6. Ogden CL, Carroll MD, Kit BK, et al. Prevalence of childhood and adult obesity in the United States. Jama. 2014;311(8):806-14.

7. Adler NE, Rehkopf DH. U.S. disparities in health: Descriptions, causes, and mechanisms. Annu Rev Public Health. 2008;29:235-52.

8. Wang YC, Gortmaker SL, Taveras EM. Trends and racial/ ethnic disparities in severe obesity among US children and adolescents. Pediatr Obes. 2011;6(1):12-20.

9. Moore LV, Dodd KW, Thompson FE, et al. Using behavioral risk factor surveillance system data to estimate the percentage of the population meeting US department of agriculture food patterns fruit and vegetable intake recommendations. Am J Epidemiol. 2015;181(12):979-88.
10. Robinson T. Applying the socio-ecological model to improving fruit and vegetable intake among low-income African Americans. J Commun Health. 2008;33(6):395-406.

11. Hughes SO, Power TG, Fisher JO, et al. Revisiting a neglected construct: Parenting styles in a child-feeding context. Appetite. 2005;44(1):83-92.

12. Anzman-Frasca S, Savage JS, Marini ME, et al. Repeated exposure and associative conditioning promote preschool children's liking of vegetables. Appetite.2012;58(2):543-53.

13. Darling N, Steinberg L. Parenting style as context: An integrative model. Psychol Bull. 1993;113(3):487.

14. Birch LL, Fisher JO, Grimm-Thomas K, et al. Confirmatory factor analysis of the child feeding questionnaire: A measure of parental attitudes, beliefs and practices about child feeding and obesity proneness. Appetite. 2001;36(3):201-10.

15. Van der Horst K, Kremers S, Ferreira I, et al. Perceived parenting style and practices and the consumption of sugarsweetened beverages by adolescents. Health Educ Res. 2006;22(2):295-304.

16. Baranowski T, Chen TA, O'Connor T, et al. Dimensions of vegetable parenting practices among preschoolers. Appetite. 2013;69:89-93.

17. O'Connor TM, Hughes SO, Watson KB, et al. Parenting practices are associated with fruit and vegetable consumption in pre-school children. Public Health Nutr. 2010;13(1):91-101.

18. Loth KA, Mac Lehose RF, Fulkerson JA, et al. Eat this, not that! parental demographic correlates of food-related parenting practices. Appetite. 2013;60:140-147.

19. Hughes SO, Anderson CB, Power TG, et al. Measuring feeding in low-income African-American and hispanic parents. Appetite. 2006;46(2):215-223.

20. Hildebrand DA, Shriver LH. A quantitative and qualitative approach to understanding fruit and vegetable availability in low-income African-American families with children enrolled in an urban head start program. J Am Die Assoc. 2010;110(5):710-18.

21. Peters RM, Aroian KJ, Flack JM. African American culture and hypertension prevention. Western J Nurs Res. 2006;28(7):831-54.

22. Bandura A,Walters RH.Social learning theory. In: Social learning theory. Englewood Cliffs, NJ: Prentice-hall. 1977;pp:1-46.

23. Dave JM, Evans AE, Condrasky MD, et al. Parent-reported social support for child's fruit and vegetable intake: Validity of measures. J Nutr Educ Behav. 2012;44(2):132-139.

24. Hughes SO, Frankel LA, Beltran AS, et al. Food parenting measurement issues: Working group consensus report. Child Obes. 2013;9(s1):S-95.

25. Zeinstra GG, Koelen MA, Kok FJ, et al. Parental childfeeding strategies in relation to Dutch children's fruit and vegetable intake. Public Health Nutr. 2010;13(6):787-796. 
26. Fusch PI, Ness LR. Are we there yet? Data saturation in qualitative research. The qualitative report. 2015;20(9):1408.

27. Fisher JO, Mitchell DC, Smiciklas-Wright H, et al. Parental influences on young girls' fruit and vegetable, micronutrient, and fat intakes. J Acad Nutr Diet. 2002;102(1):58-64.

28. Wolfenden L, Wyse RJ, Britton BI, et al. Interventions for increasing fruit and vegetable consumption in children aged 5 years and under. The Cochrane Library. 2012;11:1-58.

29. Birch LL, McPhee L, Shoba BC. What kind of exposure reduces children's food neophobia?: Looking vs. tasting. Appetite. 1987;9(3):171-8.

30. Rasmussen M, Krølner R, Klepp KI, et al. Determinants of fruit and vegetable consumption among children and adolescents: A review of the literature. Part I: Quantitative studies. Int j Behav Nutr Phy. 2006;3(1):22.

31. Blissett J. Relationships between parenting style, feeding style and feeding practices and fruit and vegetable consumption in early childhood. Appetite. 2011;57(3):826-31.

32. Vereecken C, Legtest E, De-Bourdeaudhuij I, et al. Associations between general parenting styles and specific food-related parenting practices and children's food consumption. Am J Health Promot. 2009;23(4):233-40.

33. Blissett J, Bennett C, Donohoe J, et al. Predicting successful introduction of novel fruit to preschool children. J Acad Nutr Diet. 2012;112(12):1959-67.

34. Sullivan SA, Birch LL. Pass the sugar, pass the salt: Experience dictates preference. Dev Psychol. 1990;26(4):546.

35. Bloomfield L, Kendall S. Parenting self-efficacy, parenting stress and child behaviour before and after a parenting programme. Primary Health Care Research \& Development. 2012;13(4):364-72.

36. Horodynski MA, Stommel M, Brophy-Herb H et al. Populations at risk across the lifespan: Case studies: Low-income African American and non-hispanic white mothers' self-efficacy, “picky eater" perception, and toddler fruit and vegetable consumption. Public Health Nurs. 2010;27(5):408-17.

37. Gary TL, Baptiste-Roberts K, Gregg EW, et al. Fruit, vegetable and fat intake in a population-based sample of African Americans. J Natl Med Assoc. 2004;96(12):1599.

38. Van Der Horst K. Overcoming picky eating. Eating enjoyment as a central aspect of children's eating behaviors. Appetite. 2012;58(2):567-74

39. Dovey TM, Staples PA, Gibson EL, et al. Food neophobia and 'picky/fussy'eating in children: a review. Appetite. 2008;50(3):181-193.

\section{*Correspondence to:}

Lenka H. Shriver, PhD

Department of Nutrition

School of Health and Human Sciences

University of North Carolina Greensboro

Greensboro, North Carolina, USA

Tel: 336-334-5313

E-mail: lhshrive@uncg.edu 